\title{
PRINCIPAL DENTAL OFFICERS AND DIRECTORS OF ORAL HEALTH SERVICES IN NSW AREA HEALTH SERVICES, MAY 1999
}

RESPONSIBILITY FOR ADMINISTERING DENTAL SERVICES IN AREA HEALTH SERVICES LIES WITH THE RESPECTIVE PRINCIPAL DENTAL OFFICERS AND DIRECTORS OF ORAL HEALTH SERVICES LISTED BELOW. FURTHER INFORMATION ABOUT ORAL HEALTH SERVICES IN YOUR AREA CAN BE OBTAINED FROM THE APPROPRIATE OFICER OR DIRECTOR

\begin{tabular}{|c|c|c|c|c|c|}
\hline Area Health Service & Officer or Director & Telephone & Facsimile & Email Address & Postal Address \\
\hline Central Coast & Brian Redmayne & (02) 43203278 & (02) 43202804 & bredmayne@doh.health.nsw.gov.au & $\begin{array}{l}\text { PO Box } 361 \\
\text { Gosford NSW } 2250\end{array}$ \\
\hline Central Sydney & John Wilkinson & (02) 92933228 & (02) 92933488 & Jwilko@udh3.udh.cs.nsw.gov.au & $\begin{array}{l}\text { United Dental Hospital } \\
2 \text { Chalmers St, Surry Hills NSW } 2010\end{array}$ \\
\hline Hunter & David Wright & $\begin{array}{l}(02) 49246060 \\
0407918107\end{array}$ & (02) 49246143 & DWright@doh.health.nsw.gov.au & $\begin{array}{l}\text { Dental Administrative Unit } \\
\text { PO Box 119, Wallsend NSW } 2287\end{array}$ \\
\hline Illawarra & Eva Piekarz & (02) 42971044 & (02) 42968397 & PiekarzE@doh.health.nsw.gov.au & $\begin{array}{l}\text { PO Box } 117 \\
\text { Warilla NSW } 2528\end{array}$ \\
\hline Northern Sydney & Molly Ghosh & $\begin{array}{l}(02) 99268217 \\
(02) 99266701\end{array}$ & (02) 99266735 & MGhosh@doh.health.nsw.gov.au & $\begin{array}{l}\text { Level 2, Vindin House } \\
\text { Royal North Shore Hospital } \\
\text { Pacific Hwy, St Leonards NSW } 2065\end{array}$ \\
\hline South Eastern Sydney & Anna Yoong & (02) 95525055 & (02) 95447662 & YoongA@sesahs.nsw.gov.au & $\begin{array}{l}\text { Sylvania Community Health Centre } \\
29 \text { Sylvania Rd, Sylvania NSW } 2224\end{array}$ \\
\hline South Western Sydney & Sameer Bhole & (02) 98285961 & (02) 98285977 & Sameer-Bhole2@doh.health.nsw.gov.au & $\begin{array}{l}\text { Hugh Jardine Building } \\
\text { Liverpool Hospital Eastern Campus } \\
\text { Locked Mail Bag 17, Liverpool NSW } 2170\end{array}$ \\
\hline Wentworth & Debbie McGibbon & (02) 47243380 & (02) 47242043 & Mcgibbd@wahs.health.nsw.gov.au & $\begin{array}{l}\text { Nepean Hospital Dental Clinic } \\
\text { Cnr Parker \& Derby Sts } \\
\text { Kingswood NSW } 2747\end{array}$ \\
\hline Western Sydney & Garth Dever & (02) 98457174 & (02) 98938671 & Garthd@dental.wsahs.nsw.gov.au & $\begin{array}{l}\text { Westmead Centre for Oral Health } \\
\text { Darcy Road, Westmead NSW } 2145\end{array}$ \\
\hline Far West & Michael Kowalski & $\begin{array}{l}(08) 80878925 \\
(08) 80876466\end{array}$ & $\begin{array}{l}(08) 80878927 \\
(08) 80879105\end{array}$ & MKOWALSK@doh.health.nsw.gov.au & $\begin{array}{l}\text { Broken Hill Town Clinic } \\
\text { PO Box 457, Broken Hill NSW } 2145\end{array}$ \\
\hline Greater Murray & Neville Heer & $\begin{array}{l}(02) 60581884 \\
(02) 60581800\end{array}$ & (02) 60581801 & neville.heer@gmhs.nsw.gov.au & $\begin{array}{l}\text { PO Box } 503 \\
\text { Albury NSW } 2640\end{array}$ \\
\hline Macquarie & Peter Roche & (02) 68812223 & (02) 68812230 & PROCH@doh.health.nsw.gov.au & $\begin{array}{l}\text { Po Box M61 } \\
\text { Dubbo NSW } 2830\end{array}$ \\
\hline Mid North Coast & Gordon Wainwright & $\begin{array}{l}(02) 66522295 \\
0417221514\end{array}$ & (02) 66522509 & GWainwright@doh.health.nsw.gov.au & $\begin{array}{l}\text { Locked Mail Bag } 812 \\
\text { Coffs Habour NSW } 2450\end{array}$ \\
\hline Mid Western & Richard Colgan & $\begin{array}{l}(02) 63395311 \\
(02) 63311030\end{array}$ & (02) 63311030 & & $\begin{array}{l}\text { Bathurst Base Hospital } \\
\text { Horwick St, Bathurst NSW } 2795\end{array}$ \\
\hline New England & Murray Bradford & (02) 67663341 & (02) 67665914 & & $\begin{array}{l}\text { Community Dental Services } \\
\text { Tamworth Base Hospital } \\
\text { Johnston St, Tamworth NSW } 2340\end{array}$ \\
\hline Northern Rivers & Greg Davies & (02) 66206171 & $(02) 66206216$ & GREGD@doh.health.nsw.gov.au & $\begin{array}{l}\text { Ballina Hospital Dental Clinic } \\
\text { PO Box 523, Ballina NSW } 2478\end{array}$ \\
\hline Southern & James Liaw & $\begin{array}{l}(02) 62989294 \\
018620203\end{array}$ & (02) 62991269 & JALIAW@doh.health.nsw.gov.au & $\begin{array}{l}\text { PO Box } 521 \\
\text { Queanbeyan NSW } 2620\end{array}$ \\
\hline
\end{tabular}

